\title{
Schmaler Grat der Opioide
}

\section{Bei Patienten, die wegen ihrer Schmerzen Opioide bekommen, be- steht auch ein erhöhtes Risiko, durch eine Überdosierung ums Leben zu kommen.}

- Mit zunehmendem Gebrauch von Opioiden in der Schmerztherapie in den letzten zehn Jahren stieg auch die Rate von Todesfällen durch Überdosierung dieser Medikamente massiv um $124 \%$ an. Da auf diesem Gebiet eine erhebliche Dunkelziffer der Meldungen anzunehmen ist, wurde nun die Assoziation zwischen der täglich verschriebenen Höchstdosis von Opioiden und der Einnahmeanweisung (bei Bedarf, regelmäBig oder beides) mit dem Mortalitätsrisiko durch Opioid-Überdosierung bei Patienten mit Karzinomen, chronischen Schmerzen, akuten Schmerzen und Substanzmissbrauch in einer Fall-Kohortenstudie untersucht. Patienten in Palliativ- oder Hospizeinrichtungen waren ausdrücklich ausgeschlossen.

Zwischen 2004 und 2008 erfasste man bei den Patienten der amerikanischen Veterans Health Administration 750 Fälle durch ungewollte Opioidüberdosierung und verglich sie mit einer $\mathrm{Zu}$ fallsstichprobe von 154684 Patienten, die in den Jahren 2004/2005 Opioide zur Schmerztherapie erhalten hatten. In der Beobachtungsperiode betrug die Häufigkeit einer tödlichen Überdosierung bei Personen, die mit Opioiden behandelt wurden, $0,04 \%$.

Das Risiko einer Überdosierung stand in engem Zusammenhang mit der täglich verordneten Opioidhöchstdosis. Die Hazard Ratio lag in der Gruppe von Patienten mit Verordnungen von 100 $\mathrm{mg} / \mathrm{Tag}$ oder mehr Morphin-Äquivalentdosen im Vergleich zur Gruppe mit verordneten Dosen zwischen $1 \mathrm{mg}$ und
$20 \mathrm{mg} /$ Tag bei den Substanzabhängigen bei 4,54 , bei den Patienten mit chronischen Schmerzen bei 7,18, bei akuten Schmerzzuständen bei 6,64 und bei Patienten mit Karzinomen bei 11,99. Dabei wurden Verschreibungen von Codein, Morphin, Oxycodon, Hydrocodon, Oxymorphon und Hydromorphon mit erfasst. Semisynthetische Opioide und Methadon wurden nicht berücksichtigt.

\section{Kommentar}

Es erscheint nahe liegend und fast banal, dass das Risiko von Intoxikationen, die im Extremfall tödlich verlaufen, bei Verordnung hoher Dosen von Morphin zunimmt. Dabei wurde in der Untersuchung nicht berücksichtigt, ob die Patienten auch noch aus anderen Quellen als den VHA-Apotheken Opioide bekamen. Auch das Aufheben von Tabletten durch den Patienten, möglicherweise zur Verstärkung des Effekts zu bestimmten Zeiten, als missbräuchlich genutzte Substanz oder im Extremfall sogar als Mittel zum Suizid konnte aus methodischen Gründen nicht erfasst werden. Das Problem könnte durch die Verwendung der neuen semisynthetischen Opioide sogar noch größer sein, als es sich anhand dieser Ergebnisse darstellt, da viele dieser Substanzen über transkutane Systeme appliziert werden, die ganz neue Probleme aufwerfen.

H. S. FÜESSL =

- A. S. B. Bohnert et al.

(University of Michigan Medical School, 4250 Plymouth Rd., Box 5765, Ann Arbor, Ml 48109): Association betwen Opioid Prescribing patterns and Opioide Overdose-related deaths. JAMA 305 (2011) 13, 1315-1321. 